Article

\title{
Blue and Orange Two-Color CW Laser Based on Single-Pass Second-Harmonic and Sum-Frequency Generation in MgO:PPLN
}

\author{
Dismas K. Choge ${ }^{1,2} \mathbb{1}^{\mathbb{D}}$, Huai-Xi Chen ${ }^{1}$, Yi-Bin Xu ${ }^{1}$, Lei Guo ${ }^{1}$, Guang-Wei Li ${ }^{1}$ \\ and Wan-Guo Liang ${ }^{1, *}$ \\ 1 Fujian Institute of Research on the Structure of Matter, Chinese Academy of Sciences, Fuzhou 350002, China; \\ dchoge@uoeld.ac.ke (D.K.C.); hxchen@fjirsm.ac.cn (H.-X.C.); xuyibin@fjirsm.ac.cn (Y.-B.X.); \\ guolei@fjirsm.ac.ke (L.G.); gwli@fjirsm.ac.cn (G.-W.L.) \\ 2 University of Chinese Academy of Sciences, Beijing 100049, China \\ * Correspondence: wgl@fjirsm.ac.cn; Tel.: +0591-83718125
}

Received: 19 March 2018; Accepted: 12 April 2018; Published: 18 April 2018

check for updates

Featured Application: This study used a single magnesium oxide doped periodically poled lithium niobate for the simultaneous generation of blue and orange light. The dual-wavelength source is potentially useful for biomedicine and entertainment.

\begin{abstract}
We demonstrate a compact blue and orange-two color continuous wave laser source emitting at $487 \mathrm{~nm}$ and from 597.4 to $600.3 \mathrm{~nm}$, respectively. The temperature tunable coherent orange radiation is achieved by frequency mixing $974 \mathrm{~nm}$ laser diode (LD) and a C-band amplified spontaneous emission laser source while the temperature insensitive blue radiation is generated by second-order quasi-phase-matching frequency doubling of $974 \mathrm{~nm} \mathrm{LD}$. We implement the simultaneous nonlinear processes in a single magnesium oxide doped periodically poled lithium niobate bulk crystal without the need of an aperiodic design.
\end{abstract}

Keywords: lithium niobate; second harmonic generation; sum frequency generation; quasi phase-matching; lasers

\section{Introduction}

Visible lasers have become ubiquitous in our society for numerous applications ranging from scientific research to industry and entertainment. For instance, the $488 \mathrm{~nm}$ wavelength can be matched with hundreds of fluorophores specifically tailored to excite at, or close to, $488 \mathrm{~nm}$ in life science research applications [1]. Similarly, orange lasers with wavelengths near $600 \mathrm{~nm}$ play a key role in biomedical applications [2], and laser projection displays [3]. Nonlinear conversion processes such as second harmonic generation (SHG) and sum frequency generation (SFG) are equally efficient approaches for realizing such visible light sources with periodically poled ferroelectric crystals. On the other hand, quasi-phase-matching (QPM) technique in a nonlinear optical crystal like lithium niobate (LN) provides a fascinating route towards implementation of such laser sources due to its distinct advantages which include access to the largest nonlinear coefficient and high damage threshold [4]. So far, the various approaches to implementing QPM range from uniform structures to Fibonacci optical superlattice [5], aperiodic [6], linearly chirped [7], apodized [8], and multi-segmented [9]. The non-uniform structures are in general, more suitable for broadband wavelength conversion since they provide many spatial vectors compared to uniform structures which are suited to narrowband applications. This development has led to the simultaneous generation of multiple wavelengths in the 
visible range within the same crystal which has the advantages of high brightness and large depth of focus highly suitable for laser-based color displays. Various techniques to generate red $(R)$, green $(G)$, blue (B), and even yellow $(\mathrm{Y})$ colors have been previously reported. These techniques include utilizing several crystals each for different nonlinear process [10], self-frequency doubling and self-frequency mixing in $\mathrm{Nd}^{3+}$-doped bulk aperiodically poled lithium niobate [11,12], and cascaded nonlinear interactions in aperiodically poled lithium tantalate (LT) $[13,14]$ or stoichiometric LT $[15,16]$. As an alternative to these approaches which make use of complicated domain distribution, it is desirable to continue to look for simple, compact, and efficient solutions utilizing single-pass simultaneous processes in uniformly poled structures.

Here we report the coincidental single-pass SFG and SHG phase-matching for the simultaneous generation of $\mathrm{CW}$ blue and orange light in a single magnesium oxide doped periodically poled lithium niobate (MgO:PPLN) crystal. The orange light is produced by frequency mixing $974 \mathrm{~nm}$ laser diode (LD) and a C-band amplified spontaneous emission (ASE) laser source (1525-1565 nm). The blue SHG light is generated by second-order QPM frequency doubling of $974 \mathrm{~nm}$ LD. We utilized the broadband nature of ASE for wavelength conversion into the visible range. Our device has the potential as two technologically interesting laser sources in MgO:PPLN in a simple and practical manner without the need for an aperiodic design.

\section{PPLN Design and Fabrication}

In order to realize multiple nonlinear processes in the same crystal, it is necessary to simultaneously fulfill their phase matching conditions. For SFG and SHG processes involving three interacting waves, a pump $\left(\omega_{1}\right)$, signal $\left(\omega_{2}\right)$, and an idler $\left(\omega_{3}\right)$, their respective wave vector mismatches due to material dispersion are [17]

$$
\begin{gathered}
\Delta k_{S F G}=k_{1}+k_{2}-k_{3}-2 \pi / \Lambda_{S F G} \\
\Delta k_{S H G}=k_{S H G}-2 k_{2}-2 \pi / \Lambda_{S H G}
\end{gathered}
$$

where $k_{j}=2 \pi n_{j} / \lambda_{j}$ is the wave vector at the corresponding frequency $\omega_{j}, j=1,2,3, \lambda_{j}$ is the wavelength in vacuum, $n_{j}$ is the refractive index (extraordinary) at that wavelength that can be calculated using the Sellmeier equation [18]. $\Lambda_{S F G}$ and $\Lambda_{S H G}$ are the QPM poling periods of SFG and SHG respectively given by

$$
\begin{gathered}
\frac{1}{\Lambda_{S F G}}=\frac{n_{1}}{\lambda_{1}}+\frac{n_{2}}{\lambda_{2}}-\frac{n_{3}}{\lambda_{3}} \\
\frac{1}{\Lambda_{S H G}}=\frac{n_{S H G}}{\lambda_{S H G}}-\frac{2 n_{2}}{\lambda_{2}}
\end{gathered}
$$

The two processes can occur simultaneously if $\Lambda_{S F G}=m \Lambda_{S H G}$ where $m$ is an integer. For a given material, Equations (3) and (4) can be satisfied for a specific pump and signal wavelengths and one periodicity. In this work, $\lambda_{1}$ is an LD source emitting $974 \mathrm{~nm}$ and $\lambda_{2}$ is a C-band ASE source at $1525-1565 \mathrm{~nm}$. Using standard electric poling technique, we fabricated a single period QPM device on a $1 \mathrm{~mm}$-thick z-cut MgO:LN crystal as reported in [19]. The device length is $50 \mathrm{~mm}$. To realize efficient SHG and SFG processes simultaneously, we are seeking the condition $\Delta k_{S F G}=\Delta k_{S H G}=0$. Using SNLO (AS photonics) [20], we calculated the required periodicities as $\Lambda_{S F G}=10.2 \mu \mathrm{m}$ at $60{ }^{\circ} \mathrm{C}$ and $\Lambda_{S H G}=5.1 \mu \mathrm{m}$ at $110^{\circ} \mathrm{C}$ based on Sellmeier equations with $m=2$. We fabricated the PPLN to have a period of $10.2 \mu \mathrm{m}$ for perfect quasi-phase-matched SFG and the second-order quasi-phase-matched SHG processes, and at the same time utilize the broadband nature of the ASE source. In this regard, we have coincidental phase matching for SHG of $\lambda_{1}$ but a decreased efficiency is expected because of the second-order phase matching [21]. It is worth noting that, the SHG conversion efficiency can be enhanced by a factor of 4 theoretically by using first-order QPM period. The orange laser output is obtained by frequency mixing $\lambda_{1}$ and $\lambda_{2}$ whereas the blue laser is generated by frequency doubling of $\lambda_{1}$. Theoretically, the maximum SHG power can be calculated using the relation 
$P_{S H G}=8 \pi d_{e f f}^{2} P_{1}^{2} L^{2} / n_{S H G} n_{1}^{2} \varepsilon_{0} c \lambda_{1}^{2} \omega_{f}^{2}$ [22] and the corresponding conversion efficiency is given as $\eta=P_{S H G} / P_{1}^{2}$. Similarly, the theoretical sum frequency output power can be estimated using the relation $P_{S F G}=32 \pi^{2} d_{\text {eff }}^{2} P_{1} P_{2} L / n_{S F G} \varepsilon_{0} \lambda_{S F G}^{2} c\left(n_{1} \lambda_{1}+n_{2} \lambda_{2}\right)$ [23], and the corresponding conversion efficiency can be given as $\eta=P_{S F G} / P_{1} P_{2}$, where $c$ is the speed of light in vacuum, $\varepsilon_{0}$ is the permittivity in vacuum, and $d_{e f f}=2 d_{33} / \pi$ is the effective nonlinear coefficient of MgO:PPLN. $n_{S H G}, n_{S F G}, n_{1}$, and $n_{2}$ are refractive indices at $\lambda_{S H G}, \lambda_{S F G}, \lambda_{1}$, and $\lambda_{2}$, respectively, while $\omega_{f}(\sim 43 \mu \mathrm{m})$ is the confocal beam waist. Our design is well suited for Type-0 QPM interaction $(e+e \rightarrow e)$ in MgO:PPLN in order to take advantage of the largest nonlinear coefficient. We chose MgO:LN as the nonlinear material due to its low susceptibility to optical damage photo-refraction and visible induced infrared absorption [24]. The poled crystal was then cleaned and wet etched in hydrofluoric acid (HF) to reveal the domain structure and the end faces were optically polished but have no anti-reflection coating.

\section{Experimental Setup and Output Characterization}

\subsection{Experimental Setup}

The experimental setup for the single-pass simultaneous sum frequency generation of orange laser and second harmonic generation of blue laser is illustrated in Figure 1.

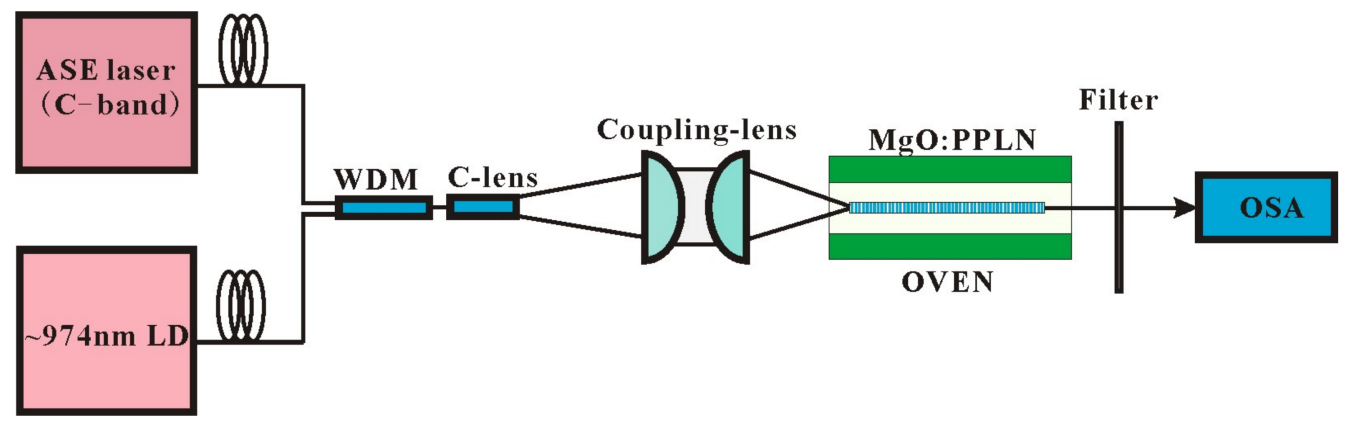

Figure 1. Schematic of the experimental setup. The beams are focused into single-pass MgO:PPLN to generate orange output by sum frequency generation (SFG) and blue output by second harmonic generation (SHG). LD: laser diode; ASE: amplified spontaneous emission; WDM: wave division multiplexer; C-lens: collimator lens; OSA: optical spectrum analyzer.

As a pump $\left(\mathrm{P}_{1}\right)$, we used a laser diode (LD) fixed at $974 \mathrm{~nm}\left(\lambda_{1}\right)$ of $0.20 \mathrm{~nm}$ spectral width, whereas the signal $\left(\mathrm{P}_{2}\right)$ was a C-band (1525-1565 nm) ASE source $\left(\lambda_{2}\right)$. A $50 \mathrm{~mm}$ long uniform MgO:PPLN was used for collinear single-pass SFG and SHG processes. The output of C-band ASE was combined with $\lambda_{1}$ inside a 50:50 wavelength division multiplexer (WDM) coupler and collimated with a C-lens before focusing at the center of the crystal using a coupling lens. We varied the temperature of the MgO:PPLN crystal to obtain the phase matching points for SFG and SHG. The temperature of the device was stabilized by a temperature controlled oven (CTL photonics) with an accuracy of $\pm 0.1^{\circ} \mathrm{C}$. The output power and bandwidth were evaluated using a laser power meter (PM100D, Thorlabs, Newton, NJ, USA) and a fiber spectrometer (BIM-6001, Brolight, Hangzhou, China).

\subsection{SFG and SHG Characterization}

The maximum input powers measured before the crystal were $\mathrm{P}_{1}=229 \mathrm{~mW}$ and $\mathrm{P}_{2}=200 \mathrm{~mW}$. Figure 2a shows the power of SHG light as a function of pump power, for an SHG wavelength of $487 \mathrm{~nm}$. The LD power, $\mathrm{P}_{1}$ was increased steadily to a maximum value of $229 \mathrm{~mW}$ by varying the operating current, and the ASE power $\mathrm{P}_{2}$ was fixed at $200 \mathrm{~mW}$ (maximum). A maximum SHG power of $5.2 \mathrm{~mW}$ was measured at $487 \mathrm{~nm}$ corresponding to $10 \% / \mathrm{W}$ conversion efficiency, lower than the expected nonlinear conversion efficiency of $\sim 29 \% / \mathrm{W}$. Figure $2 \mathrm{~b}$ illustrates the SFG power as a function of the product of pump $\left(\mathrm{P}_{1}\right)$ and signal $\left(\mathrm{P}_{2}\right)$ powers incident on the crystal. The maximum 
sum-frequency generation of $9.3 \mathrm{~mW}$ at $598.8 \mathrm{~nm}$ is achieved, resulting in an overall conversion of $\sim 20.3 \% / \mathrm{W}$, which is also lower than the expected conversion efficiency of $\sim 42 \% / \mathrm{W}$. Possible reasons for low conversion efficiencies can be attributed to an inhomogeneous temperature distribution within the crystal and/or poling imperfections in the QPM structure. As for the low SHG conversion efficiency, one predominant reason is using the second-order QPM for the given domain period $(10.2 \mu \mathrm{m})$, which can be improved by using the first-order QPM period $(5.1 \mu \mathrm{m})$. The conversion efficiencies could be further improved by using a high fundamental power.
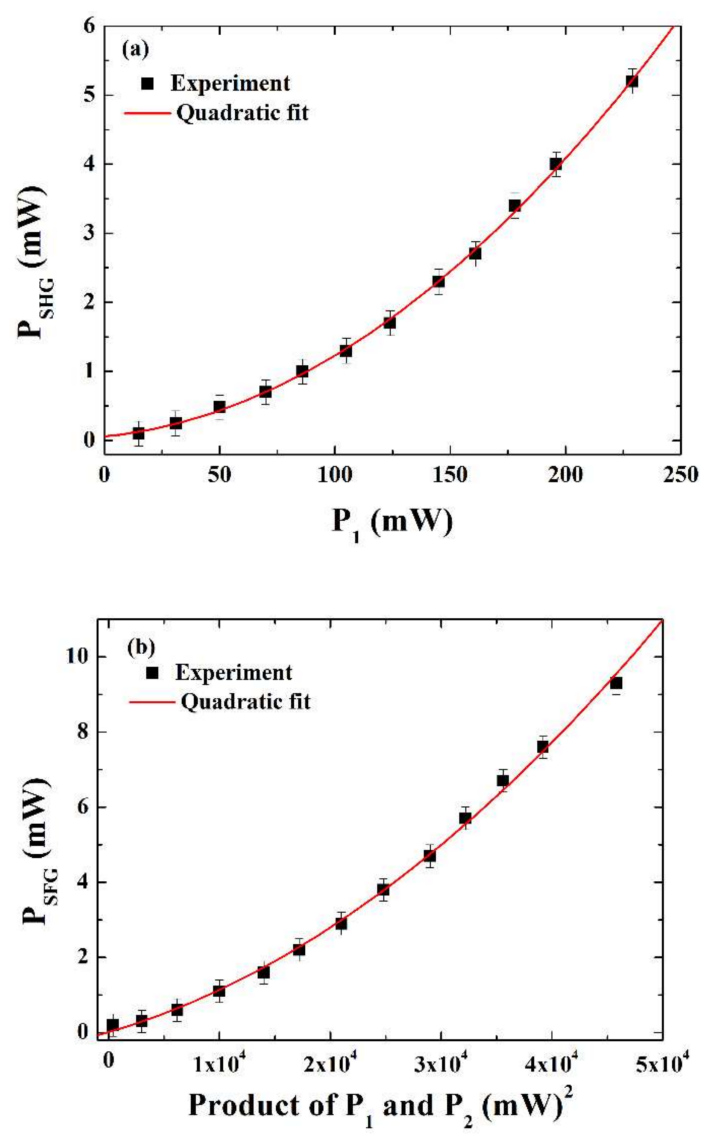

Figure 2. Measured (black squares) output power as a function of the fundamental power for (a) second harmonic generation (SHG), and (b) sum frequency generation (SFG). The solid curve in (a) corresponds to a quadratic fit to the SHG data using $P_{S H G}=\eta_{S H G} P_{1}^{2}$ and in (b) the solid curve is a quadratic fit for the SFG process using $P_{S F G}=\eta_{S F G} P_{1} P_{2}$, where $\eta_{S H G}=10 \% / \mathrm{W}$ and $\eta_{S F G}=20.3 \% / \mathrm{W}$ are the SHG and SFG normalized conversion efficiencies determined from power measurements respectively. Other fit parameters are $\mathrm{L}=50 \mathrm{~mm}$ (crystal length) and $\omega_{f}=43 \mu \mathrm{m}$. The error bars include the uncertainty in the output power measurements.

The temperature of the MgO:PPLN was varied from $25^{\circ} \mathrm{C}$ to $120^{\circ} \mathrm{C}$. Figure 3 shows the orange and blue laser intensities as a function of temperature. The SFG was always present with high intensity in the region $30-100^{\circ} \mathrm{C}$. We attributed this to the broadband nature of the ASE source used in this work. The drop in the SFG intensity below $30^{\circ} \mathrm{C}$ and above $100{ }^{\circ} \mathrm{C}$ could be because of a phase-matching condition at $\lambda_{2}$ wavelength with low intensity. A further decrease in the SFG intensity at $110{ }^{\circ} \mathrm{C}$ may be attributed to a competition between the SFG and SHG processes. The high intensity of blue light could be a result of the QPM condition for SHG satisfied at $110{ }^{\circ} \mathrm{C}$. When the temperature is set at $25^{\circ} \mathrm{C}$ (Figure $4 \mathrm{a}$, only SFG appears with relatively lower intensity. As the temperature is increased to $60^{\circ} \mathrm{C}$, the SFG intensity rises to almost twice as the intensity observed at room temperature as seen in Figure $4 \mathrm{~b}$. We attribute this to phase matching temperature of the SFG process being near $60^{\circ} \mathrm{C}$. 
There exists some competition between the SFG and SHG processes since both processes consume energy from the fundamental lasers $[25,26]$. According to Figure $4 c, d$, we can see that SHG appears along with SFG and at $110^{\circ} \mathrm{C}$, the SHG intensity reaches its maximum. We estimated a reduction of SFG power by $\sim 7 \%$ of its maximum (at $60^{\circ} \mathrm{C}$ ) when the SHG intensity is at its maximum. This phenomenon can be understood from the QPM theory that the relative intensities of nonlinear optical processes are dependent on the proximity of each process to perfect quasi-phase matching condition. The generation of blue laser is due to second-order SHG given that QPM even-order processes are possible since the average duty ratio may not be accurately 50:50 due to fabrication errors [27]. When the temperature was increased beyond $110{ }^{\circ} \mathrm{C}$, the intensity of both SHG and SFG decreased significantly as shown in Figure 3. This means that the device should be designed to operate below $110^{\circ} \mathrm{C}$ in order to generate the blue and orange lasers within the same crystal. The inset in Figure $4 \mathrm{~d}$ is a photo of the generated SHG and SFG beams.

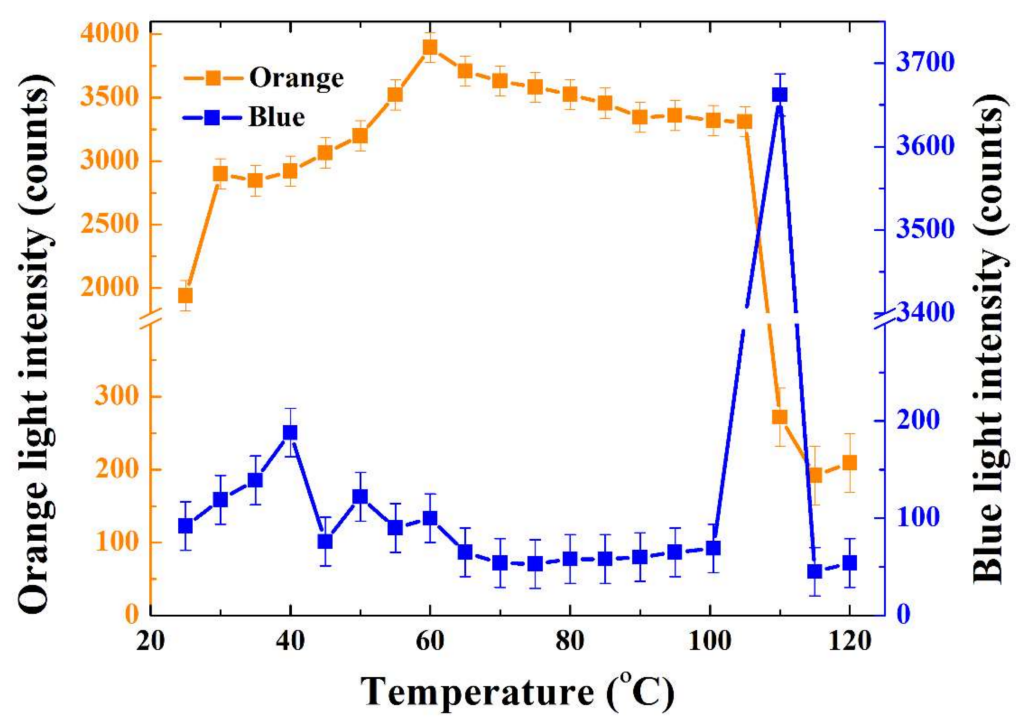

Figure 3. Intensity of SFG and SHG light as a function of temperature.
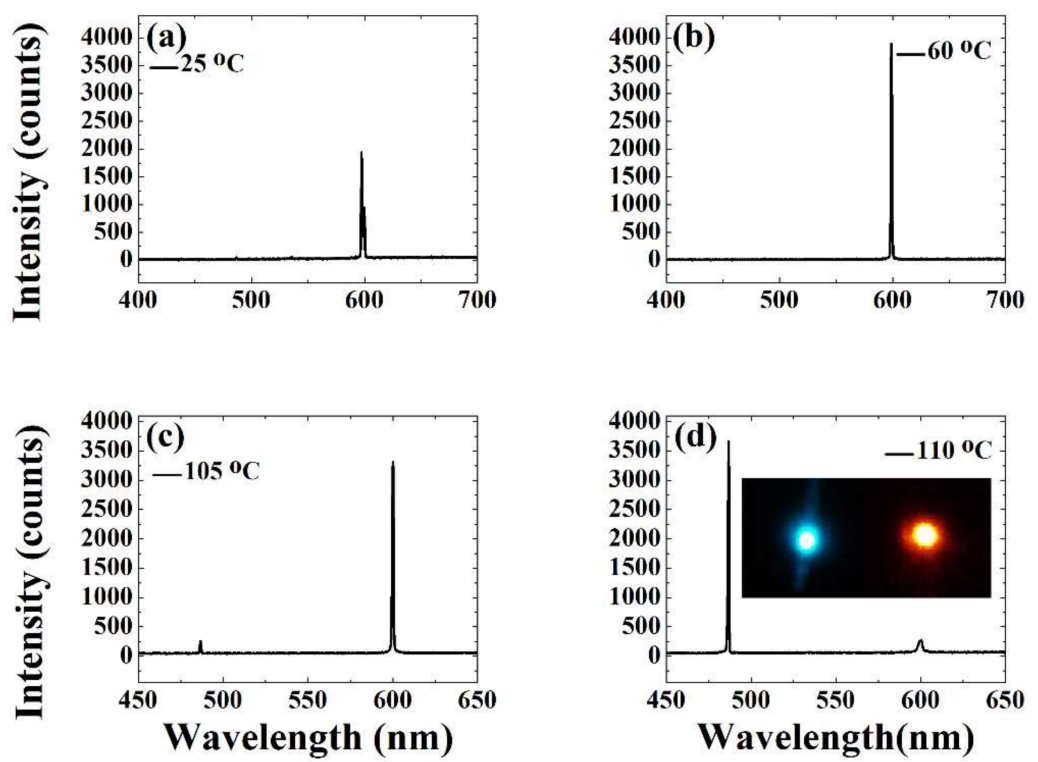

Figure 4. Normalized measured SFG and SHG output spectra at (a) $25{ }^{\circ} \mathrm{C}$; (b) $60{ }^{\circ} \mathrm{C}$; (c) $105{ }^{\circ} \mathrm{C}$; and (d) $110{ }^{\circ} \mathrm{C}$. Inset: photograph of the generated SHG and SFG beams. 
We also investigated the temperature dependence of the central wavelength of SHG and SFG. Figure 5 shows the SHG and SFG spectra at $40^{\circ} \mathrm{C}$ (black line), $60^{\circ} \mathrm{C}$ (red line) and $110^{\circ} \mathrm{C}$ (blue line) with a spectral resolution of $\sim 0.7 \mathrm{~nm}$. Whereas the central wavelength of SHG is not sensitive to the temperature variation (Figure 6), the central wavelength of SFG apparently changes by about $3 \mathrm{~nm}$ (597.4-600.3 nm) from $25^{\circ} \mathrm{C}$ to $110^{\circ} \mathrm{C}$.

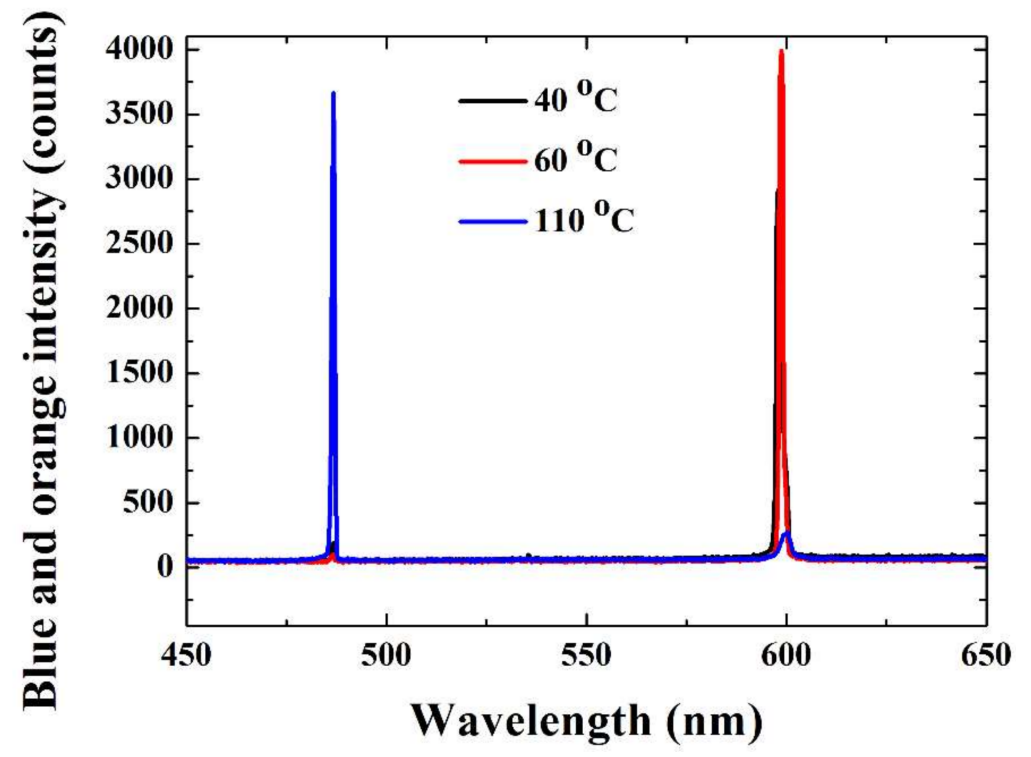

Figure 5. Measured SHG and SFG spectra at $40{ }^{\circ} \mathrm{C}$ (black), $60^{\circ} \mathrm{C}$ (red), and $110{ }^{\circ} \mathrm{C}$ (blue).

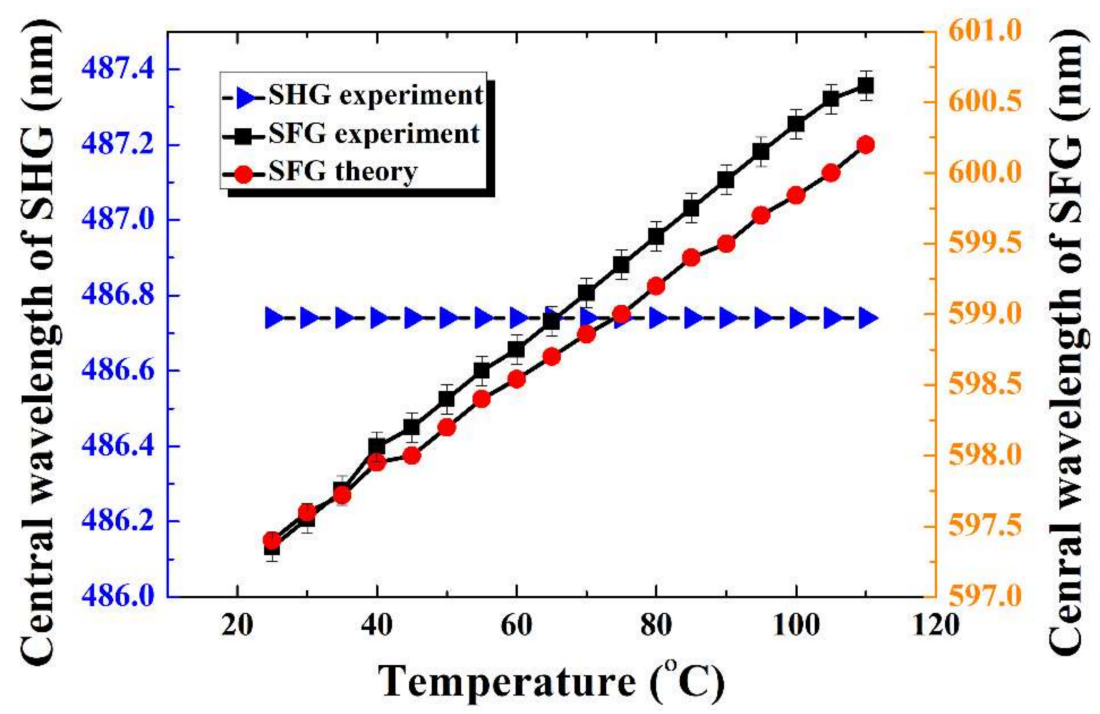

Figure 6. Dependence of central wavelengths of SHG (blue triangles) and SFG (black squares) on temperature. The red circles are calculated with the Sellmeier equation.

The shift in the central wavelength of SFG is due to the broadband nature of ASE source and the change of SFG quasi-phase-matching condition. The latter comes explicitly from the temperature dependent refractive index of MgO:PPLN according to the Sellmeier equation. In particular, the phase-matching condition shifts to longer wavelengths with an increase in temperature. According to Figure 6, the experimental data for SFG are consistent with the theoretical values calculated by using the Sellmeier equation for the same temperature range [18], demonstrating that the MgO:PPLN crystal phase-matches over the entire $50 \mathrm{~mm}$ length. 


\section{Conclusions}

We have demonstrated an approach to generate a dual-wavelength laser source based on single-pass second harmonic and sum frequency generation in a magnesium oxide doped, periodically poled lithium niobate crystal. A diode laser operating at $974 \mathrm{~nm}$ and a C-band ASE laser (1525-1565 nm) were used as the pump and signal respectively. Blue SHG light at $487 \mathrm{~nm}$ and orange SFG light tunable from $597.4 \mathrm{~nm}$ to $600.3 \mathrm{~nm}$ have been obtained by varying the temperature of the crystal from $25^{\circ} \mathrm{C}$ to $110{ }^{\circ} \mathrm{C}$. The measured central wavelength changes with a rate of $\sim 0.04 \mathrm{~nm} /{ }^{\circ} \mathrm{C}$, which can be understood from the temperature dependence of the refractive index of MgO:PPLN. We believe the dual-wavelength source will have potential applications such as in biomedicine and entertainment.

Acknowledgments: The authors gratefully acknowledge Fujian Science and Technology Service Network Initiative (STSI) Project (2016T3010).

Author Contributions: Dismas K. Choge wrote the manuscript, and conceived, designed, and performed the experiment; Dismas K. Choge and Lei Guo analyzed the experimental results; Huai-Xi Chen designed and poled the PPLN samples used in the experiment; Yi-Bin Xu and Guang-wei Li contributed in cutting, polishing, and annealing the PPLN samples; Wan-Guo Liang contributed the PPLN chips and the equipment used for preparing samples and performing experiments.

Conflicts of Interest: The authors declare no conflict of interest.

\section{References}

1. Johansson, S.; Spiekermann, S.; Wang, S.; Pasiskevicius, V.; Laurell, F.; Ekvall, K. Generation of turquoise light by sum frequency mixing of a diode-pumped solid-state laser and a laser diode in periodically poled KTP. Opt. Express 2004, 12, 4935-4940. [CrossRef] [PubMed]

2. Kapoor, V.; Subach, F.V.; Kozlov, V.G.; Grudinin, A.; Verkhusha, V.V.; Telford, W.G. New lasers for flow cytometry: Filling the gaps. Nat. Methods 2007, 4, 678-679. [CrossRef] [PubMed]

3. Xu, Z.Y.; Bi, Y. Large laser projection displays utilizing all-solid-state RGB lasers. In Light-Emitting Diode Materials and Devices; SPIE Proceedings; John Wiley \&Sons Ltd.: Oboken, NJ, USA, 2005; Volume 5632, pp. 115-122.

4. Mizuuchi, K.; Yamamoto, K.; Kato, M. Harmonic blue light generation in X-cut $\mathrm{MgO}: \mathrm{LiNbO}_{3}$ waveguide. Electron. Lett. 1997, 33, 806-807. [CrossRef]

5. Zhu, S.N.; Zhu, Y.Y.; Ming, N.B. Quasi-phase-matched third-harmonic generation in a quasi-periodic optical superlattice. Science 1997, 278, 843-846. [CrossRef]

6. Liu, X.; Zhang, H.; Guo, Y.; Li, Y. Optimal Design and Applications for Quasi-Phase-Matching Three-Wave Mixing. IEEE J. Quantum Electron. 2002, 38, 1225-1233.

7. Charbonneau-Lefort, M.; Fejer, M.M.; Afeyan, B. Tandem chirped quasi-phase-matching grating optical parametric amplifier design for simultaneous group delay and gain control. Opt. Lett. 2005, 30, 634-636. [CrossRef] [PubMed]

8. Umeki, T.; Asobe, M.; Yanagawa, T.; Tadanaga, O.; Nishida, Y.; Magari, K.; Suzuki, H. Broadband wavelength conversion based on apodized $\chi^{2}$ grating. J. Opt. Soc. Am. B 2009, 26, 2315-2322. [CrossRef]

9. Zeng, X.; Ashihara, S.; Wang, Z.; Wang, T.; Chen, Y.; Cha, M. Excitation of two-colored temporal solitons in a segmented quasi-phase-matching structure. Opt. Express 2009, 17, 16877-16884. [CrossRef] [PubMed]

10. Brunner, F.; Innerhofer, E.; Marchese, S.V.; Südmeyer, T.; Paschotta, R.; Usami, T.; Ito, H.; Kurimura, S.; Kitamura, K.; Arisholm, G.; et al. Powerful red-green-blue laser source pumped with a mode-locked thin disk laser. Opt. Lett. 2004, 29, 1921-1923. [CrossRef] [PubMed]

11. Capmany, J. Simultaneous generation of red, green, and blue continuous-wave laser radiation in $\mathrm{Nd}^{3+}$-doped aperiodically poled lithium niobate. Appl. Phys. Lett. 2001, 78, 144-146. [CrossRef]

12. Capmany, J.; Bermúdez, V.; Callejo, D.; García Solé, J.; Diéguez, E. Continuous wave simultaneous multi-self-frequency conversion in $\mathrm{Nd}^{3+}$-doped aperiodically poled bulk lithium niobate. Appl. Phys. Lett. 2000, 76, 1225-1227. [CrossRef]

13. Liao, J.; He, J.L.; Liu, H.; Du, J.; Xu, F.; Wang, H.T.; Zhu, S.N.; Zhu, Y.Y.; Ming, N.B. Red, yellow, green and blue-Four-color light from a single, aperiodically poled $\mathrm{LiTaO}_{3}$ crystal. Appl. Phys. B Lasers Opt. 2004, 78, 265-267. [CrossRef] 
14. Liao, J.; He, J.L.; Liu, H.; Wang, H.T.; Zhu, S.N.; Zhu, Y.Y.; Ming, N.B. Simultaneous generation of red, green, and blue quasi-continuous-wave coherent radiation based on multiple quasi-phase-matched interactions from a single, aperiodically-poled $\mathrm{LiTaO}_{3}$. Appl. Phys. Lett. 2003, 82, 3159-3161. [CrossRef]

15. Gao, Z.D.; Zhu, S.N.; Tu, S.Y.; Kung, A.H. Monolithic red-green-blue laser light source based on cascaded wavelength conversion in periodically poled stoichiometric lithium tantalate. Appl. Phys. Lett. 2006, 89, 181101. [CrossRef]

16. Xu, P.; Zhao, L.N.; Lv, X.J.; Lu, J.; Yuan, Y.; Zhao, G.; Zhu, S.N. Compact high-power red-green-blue laser light source generation from a single lithium tantalate with cascaded domain modulation. Opt. Express 2009, 17, 9509. [CrossRef] [PubMed]

17. Baker, K.L. Single-pass gain in a chirped quasi-phase-matched optical parametric oscillator. Appl. Phys. Lett. 2003, 82, 3841-3843. [CrossRef]

18. Gayer, O.; Sacks, Z.; Galun, E.; Arie, A. Temperature and wavelength dependent refractive index equations for MgO-doped congruent and stoichiometric $\mathrm{LiNbO}_{3}$. Appl. Phys. B Lasers Opt. 2008, 91, 343-348. [CrossRef]

19. Chen, B.Q.; Ren, M.L.; Liu, R.J.; Zhang, C.; Sheng, Y.; Ma, B.Q.; Li, Z.Y. Simultaneous broadband generation of second and third harmonics from chirped nonlinear photonic crystals. Light Sci. Appl. 2014, 3, 1-6. [CrossRef]

20. Smith, A. SNLO Version 6.7 Nonlinear Optics Software. Available online: http://www.as-photonics.com/ snlo (accessed on 27 March 2017).

21. Yu, N.E.; Kurimura, S.; Kitamura, K. Higher-Order Quasi-Phase Matched Second Harmonic Generation in Periodically Poled MgO-Doped Stoichiometric $\mathrm{LiTaO}_{3}$. J. Korean Phys. Soc. 2005, 47, 636-639. [CrossRef]

22. Bostani, A.; Ahlawat, M.; Tehranchi, A.; Morandotti, R.; Kashyap, R. Design, fabrication and characterization of a specially apodized chirped grating for reciprocal second harmonic generation. Opt. Express 2015, 23, 5183-5189. [CrossRef] [PubMed]

23. Powers, P.E. Field Guide to Nonlinear Optics; Greivenkamp, J.E., Ed.; SPIE: Bellingham, WA, USA, 2013.

24. Georgiev, D.; Gapontsev, V.P. Watts-level frequency doubling of a narrow line linearly polarized Raman fiber laser to $589 \mathrm{~nm}$. Opt. Express 2005, 13, 6772-6776. [CrossRef] [PubMed]

25. Dang, W.; Chen, Y.; Gong, M.; Chen, X. Competition between SFG and two SHG in broadband type-I QPM. Appl. Phys. B Lasers Opt. 2013, 110, 477-482. [CrossRef]

26. Mehendale, S.C.; Forysiak, W.; Cheng, C.; Harrison, R.G. Influence of competition on optical sum frequency generation. Opt. Commun. 1986, 59, 304-308. [CrossRef]

27. Fejer, M.M.; Magel, G.A.; Jundt, D.H.; Byer, R.L. Quasi-Phase-Matched Second Harmonic Generation: Tuning and Tolerances. IEEE J. Quantum Electron. 1992, 28, 2631-2654. [CrossRef] 